\title{
Purulent Appendicular Peritonitis in Pregnancy: Management and Pregnancy Outcome
}

\author{
Piazze Juan ${ }^{1,4 *}$, Donfrancesco Cristina ${ }^{1,2}$, Testani Costantino ${ }^{1}$ and Rea Silvio ${ }^{3,4}$ \\ ${ }^{1}$ Department of Gynecology and Obstetrics, SS. Trinity Hospital, Italy \\ ${ }^{2}$ Department of Experimental Medicine, Sapienza University of Rome, Italy \\ ${ }^{3}$ Oncological Surgery, University of L'Aquila, Italy \\ ${ }^{4}$ Carlo Ferri Foundation, Monterotondo, Italy
}

*Corresponding author: Juan Piazze, Department of Gynecology and Obstetrics, SS.

Trinity Hospital, Carlo Ferri Foundation, Monterotondo, Italy.

\begin{abstract}
Appendicitis represents the most common cause of acute abdomen in pregnancy, but diagnosis remains challenging.

Case presentation: A 40-years woman at 23 weeks of pregnancy with an under-head presentation of purulent peritonitis secondary to acute appendicitis, we present this case report in order to describe the uneventful maternal-neonatal outcome at term, despite the risky acute abdomen caused by a retrocecal appendicitis at mid pregnancy.
\end{abstract}

Keywords: Pregnancy, appendicitis, acute abdominal pain, peritonitis, cesarean section

\section{Background}

Appendicitis represents the most common cause of acute abdomen in pregnancy, accounting for $25 \%$ of non-obstetric surgery in pregnant women, with an incidence between $0.06 \%$ and $0.28 \%[1,2]$. Forty per cent of cases takes place on the second trimester [3]. Diagnosis of appendicitis during pregnancy remains challenging, for the low sensitivity of ultrasound (US) and low predict value of typical symptoms, due to the change of appendix location and to the physiological mild leukocytosis [4-7]. Therefore, complications occur more frequently during pregnancy than in non-pregnant women [8].

In the current case we report our experience of the challenging diagnosis and subsequent laparotomic treatment of purulent appendicular peritonitis in the second trimester, reflecting an optimal management of this threatening situation.

\section{Case Presentation}

A gravida 2, para 2, 40-year-old white woman at 23 weeks of gestation, presented to the emergency room of our Hospital with abdominal discomfort and gastrointestinal symptoms such as nausea and vomiting. At the time of admission to hospital, her blood pressure was $110 / 70 \mathrm{mmHg}$, heart-frequency $72 \mathrm{bpm}$, body temperature $37^{\circ} \mathrm{C}$ and laboratory tests indicated a mild leukocytosis with neutrophilia, low monocytes and lymphocytes. The local examination showed a regular vagina without pathological loss, a posterior cervix and a soft uterine body. On abdominal examination she presented right back pain; Giordano's test was positive at the same side. At ultrasound evaluation, she presented dilatation of the right renal pelvis and proximal ureter, and palpable inguinal lymph nodes at the same side, as well as good fetal parameters. She was hospitalized with the diagnosis of right renal colic. A treatment with antibiotics, antiemetics and antalgic drugs was started. The day after admission her conditions get worse, and she presented temperature $\left(37.8^{\circ} \mathrm{C}\right)$. This time abdominal US showed moderate fluid in all quadrants.

So, the patient was taken for emergency exploratory laparotomy. We decided to perform laparotomy in consideration of her two previous cesarean section and the possible difficulties we could have found due to adhesions. Intraoperatively we found out a diffuse purulent peritonitis secondary to acute appendicitis. Appendix was even dislocated in the retrocecal site. Appendicectomy and thorough abdominal washing were performed. The postoperative course was normal. The patient's symptoms regressed. She was 
treated with large spectrum antibiotics for seven days. Histological examination found acute purulent appendicitis.

The patient was discharged on the 8th day after surgery and was constantly followed by our Division. At 39 weeks of pregnancy she underwent cesarean section. This time we found a clean abdominal cavity, without signs of inflammations or adhesions. A healthy female child, weighting 3.300 gr, was born. The surgery was uneventful, the postoperative course and her blood exams were normal. The patient and her baby were discharged home three days after cesarean section in healthy conditions.

\section{Comment}

In cases such as the one of the present case report diagnosis may be even more difficult. Appendicular compromise should be kept in mind when a pregnant woman in which appendix is dislocated in the retrocecal site, presents with an unclear back pain. In these patient's decisions about surgery become more challenging, because most of the signs of appendicitis as pain or hyperemesis are also found during normal pregnancy period. Surgery is usually unavoidable, but what type of technique, laparoscopy or laparotomy has to be used, is still debated. Laparoscopic surgery for pregnant women is usually considered safe, with low rate of intraoperative complications in all trimesters [9], but it is associated with significantly higher rate of fetal loss compared to laparotomic appendectomy [10].

\section{Acknowledgement}

To the midwives Mrs Maria Pia Arcese and Mrs Tina Facchini for their collaboration and logistic support.

\section{Conflict of Interest}

No conflict of interest.

\section{References}

1. Andersen B, Nielsen TF (1999) Appendicitis in pregnancy: diagnosis, management and complications. Acta Obstet Gynecol Scand 78(9): 758762.

2. Tika Ram Bhandari, Sudha Shahi, Sarita Acharya (2017) Acute appendicitis in pregnancy and the developing world. Int Sch Res Notices 2017: 2636759.

3. Barber-Millet S, Bueno Lledó J, Granero Castro P, Gómez Gavara I, Ballester Pla N, et al. (2016) Update on the management of non-obstetric acute abdomen in pregnant patients. Cir Esp 94(5): 257-265.

4. Abbasi N, Patenaude V, Abenhaim HA (2014) Evaluation of obstetrical and fetal outcomes in pregnancies complicated by acute appendicitis. Arch Gynecol Obstet 290(4): 661-667.

5. Spalluto LB, Woodfield CA, DeBenedectis CM, Lazarus E (2012) MR imaging evaluation of abdominal pain during pregnancy: appendicitis and other nonobstetric causes. Radiographics 32(2): 317-334.

6. Pitkin RM, Witte DL (1979) Platelet and leukocyte counts in pregnancy. JAMA 242(24): 2696-2698.

7. Greenhalgh R, Punwani S, Taylor SA (2008) Is MRI routinely indicated in pregnant patients with suspected appendicitis after equivocal ultrasound examination? Abdom Imaging 33(1): 21-25.

8. Rizzo AG (2003) Laparoscopic surgery in pregnancy: long-term followup. J Laparoendosc Adv Surg Tech A 13(1): 11-15.

9. Walsh CA, Tang T, Walsh SR (2008) Laparoscopic versus open appendicectomy in pregnancy: a systematic review. Int J Surg 6(4): 339344.

10. C Palanivelu, M Rangarajan, R Parthasarathi (2006) Laparoscopic Appendectomy in Pregnancy: a Case Series of Seven Patients. JSLS 10(3): 321-325. 